\title{
Editorial
}

\section{Footnotes to Kraepelin: changes in the classification of mood disorders with DSM-5}

Nicola J. Kalk and Allan H. Young

\section{Summary}

Reliable diagnosis of mood disorders continues to pose a challenge. This is surprising because they have been recognised clinically since classical times. Mood disorders are also common: major depressive disorder affects nearly 300 million people worldwide and bipolar affective disorder nearly 60 million and they are a major cause of disability. Nonetheless, the reliability trials of the updated Diagnostic and Statistical Manual, Fifth Edition (DSM-5) found that the reliability of the diagnosis of major depressive disorder was in the 'questionable' range. Although the reliability of the diagnosis of bipolar I disorder in the same trials was 'good', the sample size of the individuals recruited to validate bipolar II disorder was insufficient to confirm reliability. As the epidemiological prevalences of bipolar I and bipolar II disorders are the same, this alone implies problems in its recognition. Here, we critically evaluate the most recent iteration of DSM mood disorder diagnoses in a historical light and set out the implications for clinical practice and research.

\section{Declaration of interest}

N.J.K. has attended educational activities funded by GlaxoSmithKline (GSK) and by Lundbeck and has worked on data from a study funded by Wyeth; her PhD was jointly funded by the Wellcome Trust and GSK. A.H.Y. has given paid lectures and is on advisory boards for the following companies with drugs used in affective and related disorders: Astrazenaca, Eli Lilly, Janssen, Lundeck, Sunovion, Servier, Livanova. He is Lead Investigator for the Embolden Study (Astrazenaca), $\mathrm{BCl}$ Neuroplasticity study and Aripiprazole Mania Study, which are investigator-initiated studies from Astrazenaca, Eli Lilly, Lundbeck, and wyeth.

\section{Copyright and usage}

(C) The Royal College of Psychiatrists 2017. This is an open access article distributed under the terms of the Creative Commons Non-Commercial, No Derivatives (CC BY-NC-ND) license.
Nicola J. Kalk is a clinical lecturer at the National Addictions Centre, King's college London, interested in comorbidity of addiction and mood disorders, comorbidity of alcohol and opiate dependence, and developing new treatments to target the harms of drugs and alcohol. Allan $\mathrm{H}$. Young is a professor in the Department of Psychological Medicine, Institute of Psychiatry, Psychology and Neuroscience, King's College London. His main interests are the cause and treatments for severe psychiatric illnesses, particularly mood disorders.

Diagnosis of mood disorders continues to pose a challenge. This is surprising because they have been recognised clinically since classical times: Hippocrates in $400 \mathrm{BC}$ and Aretaeus of Cappadocia in the second century $\mathrm{AD}$ clearly described mood disorders. ${ }^{1,2}$ Mood disorders are also common: at any one time major depressive disorder affects nearly 300 million people worldwide and bipolar affective disorder nearly 60 million. ${ }^{3}$ Finally, they are a major cause of disability: major depressive disorder is the second leading cause of years lost to disability accounting for $8.2 \%$ of the global burden. ${ }^{4}$ Nonetheless, the reliability trials of the updated Diagnostic and Statistical Manual, Fifth Edition (DSM-5), which codifies the diagnostic criteria of mood disorder and is the main diagnostic system used in psychiatric research, ${ }^{5}$ found that the reliability of the diagnosis of major depressive disorder was in the 'questionable' range. This implies that experienced clinicians could be expected to agree about the diagnosis only $15 \%$ more frequently than by chance alone. ${ }^{6,7}$ Although the reliability of the diagnosis of bipolar I disorder in the same trials was good, the sample size of the individuals recruited to validate bipolar II disorder was insufficient to confirm reliability. Given that the epidemiological prevalence of bipolar II disorder is the same as that of bipolar I disorder, this alone implies problems in its recognition. Here, we set out to describe the development of diagnoses of mood disorders, critically evaluate the most recent iteration of the DSM in a historical light and set out the implications for clinical practice and research.

Modern classification of mood disorder dates from the 19th century in Europe and continues to be guided by the ideas of Emil Kraepelin. Although he built on the recognition of a syndrome of alternating mania and melancholia identified as hereditary by Esquirol in $1838,{ }^{2}$ named folie circulaire by Falret earlier in $1851,{ }^{8}$ Kraepelin's contribution was distinctive. In contrast to Freud, who was born in the same year, he believed that mental disorders should be characterised according to clinical presentation and natural history, rather than unproven theories about causation. He gathered longitudinal clinical data from over 8000 individuals as a basis for his classification, yielding a detailed description of the clinical variety of mood disorder. ${ }^{8}$ Kraepelin came to the conclusion that all mood disorders comprised a spectrum, encompassing bipolar affective disorder, depressive disorder, and instability of mood as a function of personality, as representative of the same underlying disturbances in affect, cognition and motivation. He classified different 'mixed' states consequently according to disturbances of each of these aspects, for example, distinguishing depressive or anxious mania from mania with poverty of thought. ${ }^{8}$ Similarly, he made the observation that it was "fundamentally and practically quite impossible to keep apart in any consistent way, simple, periodic and circular cases; everywhere there are gradual transitions' (Kraepelin, p. 2 1920). ${ }^{8}$ The $1 \%$ conversion rate per year from major depressive disorder to bipolar I disorder, and a similar conversion rate for bipolar II disorder, which remains constant throughout its course, supports his opinion. ${ }^{9}$

During the early part of the 20th century, there was little comparison between various classifications, so the reliability of diagnosis was untested. ${ }^{10}$ This was - and continues to be - of fundamental importance, as reliability is the only available test of the 'goodness' of psychiatric diagnosis. The validity of psychiatric diagnosis could not (and as yet cannot) be tested, as there is no objective clinical investigation leading to a definitive diagnosis, in the way, for example, that a liver biopsy may produce evidence of cirrhosis. At the same time, diagnosis was complicated by the dominance of psychoanalysis, which formulated mental disorders in terms of underlying intrapsychic conflicts and did not emphasise categorical distinctions. ${ }^{10}$ This state of affairs left psychiatry vulnerable to philosophical attacks inside and outside the field, 
(e.g. by Szasz ${ }^{11}$ ). More pragmatically, financial pressures from third-party funders of healthcare, and public schemes such as Medicaid in the USA, also functioned uneasily alongside a psychiatric framework that lacked clear categorical distinctions. ${ }^{10}$

The precipitant for greater standardisation of diagnosis came from the USA-UK Diagnostic Project undertaken by, among others, Robert Kendell and Robert Spitzer in the 1960s, which confirmed that psychiatric diagnosis was not reliable between countries. The study followed debate regarding the prevalence in the two countries for schizophrenia and bipolar disorder: admissions for schizophrenia were $50 \%$ more common in the USA, whereas admissions for bipolar depression were nine times higher in England and Wales (Kramer, 1961 in Kendell ${ }^{12}$ ). To resolve this, it had to be established that criteria for diagnosis were similar. What emerged from the US-UK Diagnostic Project was that if criteria for diagnosis were harmonised, the prevalence of schizophrenia and bipolar disorder was the same in both countries. ${ }^{12}$ This study laid the groundwork for the development of the third revision of Diagnostic and Statistical Manual of Mental Disorders (DSM-III) in the USA and the chapter on mental and behavioural disorders in the International Classification of Disease (ICD) produced by the World Health Organization. Around the same time, Eli Robbins, Sam Guze and colleagues at Washington University School of Medicine in St Louis broke with the psychodynamic hegemony, publishing diagnostic criteria for psychiatric conditions based on symptoms and signs, clinical course and heredity followed by a seminal text on psychiatric diagnosis. ${ }^{13,14}$ Robert Spitzer, who chaired the DSM-III taskforce, was influenced by this group of 'neo-Kraepelinians' and used their evolving research evidence regarding clinical symptoms and signs as well as longitudinal prognosis, rather than unproven explanatory frameworks, to develop the classification system. ${ }^{10}$ The DSM was subsequently revised in 1987, 1994 and most recently, 2013.

The strengths of the DSM approach, and that of the ICD-10, which was influenced by it, is that the classification has proven to be stable cross-culturally that there is evidence of heritability of disorders as defined by DSM-III and DSM-IV and that the described entities have shown diagnostic stability over time. ${ }^{5}$ The weaknesses of the system relate to observations that symptoms, and treatment response to particular medications, do not respect the boundaries of classification thereby raising the possibility that the defined categories either encompass heterogenous disorders, represent pleiotrophic manifestations of the same process, or some combination of the two.

The most recent edition - DSM-5 - has been criticised, by, among others, Dr Frances, the head of the DSM-IV task force, as an unnecessary endeavour. ${ }^{15,16}$ In part, this related to the expectation - which has not been met - that the substantial advances in neuroscience and genetics in the intervening years, supported by substantial research funding, would lead to a paradigm shift in classification related to underlying pathological processes that could be objectively assessed. The Research Domain Criteria (RDoC) championed by Dr Thomas Insel of the National Institute for Mental Health in 2010 made an ambitious attempt at this by defining basic dimensions of brain function - such as fear circuitry - which could be analysed from genes to neural circuits to behaviour and used as a basis for a multiaxial classification system. ${ }^{17}$ However, these developments were not considered sufficiently robust or aligned to stable clinical entities to allow integration into the DSM-5, with its attendant implications for healthcare delivery and insurance remuneration. ${ }^{5}$

Thus, the DSM- 5 represents incremental changes to the DSMIII and DSM-IV. It may not have been necessary, but it seems that some of the refinements are useful. The inclusion of increased activity or energy as a core symptom of mood elevation may prove useful in clarifying the diagnosis of mania or hypomania and lends itself to augmented monitoring by smart devices, such as actigraphy, to improve the specificity of diagnosis. Other changes with promising potential include the additional specifiers that can be coded for both bipolar affective disorder and major depressive disorder. The specifier 'with mixed features' has replaced the category of 'bipolar disorder, mixed', and the criteria have changed. DSM-IV required that the individual met full criteria for both depression and mania, with the consequence that mixed mood states were underdiagnosed and inappropriately treated as unipolar depression. ${ }^{18}$ The prevalence of depressive symptoms, which occurs in around one-third of people with mania, ${ }^{19}$ was also not recognised. The addition of an anxious distress specifier also relates to important clinically observed variability and is likely to diminish artificial inflation of comorbidity. Thus, the addition of specifiers more closely reflects Kraepelin's view of the spectrum of alterations in mood, volition and thought that could occur during an episode of mood disorder. Further, it has the potential to stratify research to identify more homogeneous subgroups of individuals, to develop more personalised treatment. This approach has already proven useful: lurasidone, an atypical antipsychotic, has been found to be effective, not only for people with bipolar depression, but also for people with major depressive disorder with mixed features. ${ }^{20} \mathrm{~A}$ more controversial, but potentially useful change is the removal of bereavement as an exclusion criterion for depression. Although this has been criticised in some quarters as pathologising a normal experience, ${ }^{15}$ bereavement, like other losses, can precipitate a depressive episode and it could be equally argued that this change will allow people to access appropriate treatment.

The separation of bipolar disorder and depression into separate chapters is potentially more problematic. The authors argue that the rationale for placing bipolar disorder as a distinct chapter between those on schizophrenia and depression was that it is 'a bridge between the two diagnostic classes in terms of symptomatology, family history and genetics'. ${ }^{21}$ Thus, major depressive disorder with subthreshold manic features - so-called mixed features - is in an entirely separate chapter to other bipolar spectrum conditions, despite the assumed elevated risk of conversion to bipolar disorder in this group. This seems counterintuitive given that one of the goals of classification is to provide stable diagnoses. ${ }^{16}$

In summary, the contemporary classification of mood disorders outlined in the DSM-5 remains a system conceived in the age of steam. The addition of specifiers in particular recalls Kraepelin's conception of a spectrum of mood disorders. Although Kraepelin did not believe that the spectrum was clinically useful, the application of specifiers to major depressive disorder in particular may result in delineation of subgroups with improved diagnostic reliability and differential treatment response. Developments such as $\mathrm{RDoC}$, which attempt to integrate developing understanding of neurobiology into classification, are complementary rather than replacements at this stage. The DSM- 5 and ICD-10 systems remain essential to clinicians, to facilitate communication and allow research to proceed. We await future developments.

\footnotetext{
Nicola J. Kalk, MBChB, PhD, MRCPsych, South London and Maudsley NHS Foundation Trust, London, UK, and National Addictions Centre, Institute of Psychiatry, Psychology and Neuroscience, King's College London, London, UK; Allan H. Young, MBChB, MPhil, PhD, FRCPsych, FRCPC, South London and Maudsley NHS Foundation Trust London, UK, and Department of Psychological Medicine, Institute of Psychiatry, Psychology and Neuroscience, King's College London, London, UK

Correspondence: Nicola J. Kalk, National Addictions Centre, King's College London, 4 Windsor Walk, Denmark Hill, London SE5 8BB, UK. Email: nicola.kalk@kcl.ac.uk

First received 10 Feb 2017, final revision 4 Apr 2017, accepted 9 Apr 2017
} 


\section{Acknowledgements}

This article represents independent research funded by the National Institute for Health Research (NIHR) Biomedical Research Centre at South London and Maudsley NHS Foundation Trust and King's College London. The views expressed are those of the authors and not necessarily those of the NHS, the NIHR or the Department of Health.

\section{References}

1 Angst J, Marneros A. Bipolarity from ancient to modern times: conception, birth and rebirth. J Affect Disord 2001; 67: 3-19.

2 Kaufman KR. The ups and downs of bipolar disorder. Ann Clin Psychiatry 2003; 15 $81-4$

3 Whiteford HA, Ferrari AJ, Degenhardt L, Feigin V, Vos T. The global burden of mental, neurological and substance use disorders: an analysis from the Globa Burden of Disease Study 2010. PLOS One 2015; 10: e0116820.

4 Ferrari AJ, Charlson FJ, Norman RE, Patten SB, Freedman G, Murray CJ, et al. Burden of depressive disorders by country, sex, age, and year: findings from the Global Burden of Disease Study 2010. PLOS Med 2013; 10: e1001547.

5 Hyman SE. Can neuroscience be integrated into the DSM-V? Nat Rev Neurosci 2007; 8: 725-32.

6 Regier DA, Narrow WE, Clarke DE, Kraemer HC, Kuramoto SJ, Kuhl EA, et al. DSM-5 field trials in the United States and Canada, Part II: test-retest reliability of selected categorical diagnoses. Am J Psychiatry 2013; 170: 59-70.

7 Fleiss JL, Spitzer RL, Endicott J, Cohen J. Quantification of agreement in multiple psychiatric diagnosis. Arch Gen Psychiatry 1972; 26: 168-71.

8 Zivanovic $\mathrm{O}$, Nedic A. Kraepelin's concept of manic-depressive insanity: one hundred years later. J Affect Disord 2012; 137: 15-24.

9 Angst J, Sellaro R, Stassen HH, Gamma A. Diagnostic conversion from depression to bipolar disorders: results of a long-term prospective study of hospital admissions. J Affect Disord 2005; 84: 149-57.
10 Mayes R, Horwitz AV. DSM-III and the revolution in the classification of mental illness. J Hist Behav Sci 2005; 41: 249-67.

11 Szasz T. The Myth of Mental Illness: Foundations of a Theory of Personal Conduct. Harper and Row, 1961.

12 Kendell RE. The Role of Diagnosis in Psychiatry. Blackwell Scientific, 1975

13 Feighner JP, Robins E, Guze SB, Woodruff RA Jr., Winokur G, Munoz R. Diagnostic criteria for use in psychiatric research. Arch Gen Psychiatry 1972; 26: 57-63.

14 Woodruff RA, Goodwin DW, Guze SB. Psychiatric Diagnosis. Oxford University Press, 1974.

15 Strakowski SM, Frances AJ. What's wrong with DSM-5? Medscape Psychiatry 1 June 2012.

16 Strakowski SM. Do we need a DSM-V? SOc Biol Psychiatry Newslett October 2011.

17 Insel $T$, Cuthbert B, Garvey M, Heinssen R, Pine DS, Quinn K, et al. Research domain criteria (RDOC): toward a new classification framework for research on mental disorders. Am J Psychiatry 2010; 167: 748-51.

18 Tandon R. Mood disorders in DSM-5: best diagnoses today and a bridge to tomorrow. Asian J Psychiatry 2013; 6: 279-80.

19 Young $\mathrm{AH}$, Eberhard J. Evaluating depressive symptoms in mania: a naturalistic study of patients with bipolar disorder. Neuropsychiatr Dis Treat 2015; 11 1137-43.

20 Suppes T, Silva R, Cucchiaro J, Mao Y, Targum S, Streicher C, et al. Lurasidone for the treatment of major depressive disorder with mixed features: a randomized, double-blind, placebo-controlled study. Am J Psychiatry 2016; 173: 400-7.

21 American Psychiatric Association. Diagnostic and Statistical Manual of Mental Disorders, 5th edn (DSM-5). APA, 2013. 